\title{
POLÍNIAS COSTEIRAS DA ANTÁRTICA ATRAVÉS DE UMA REANÁLISE OCEÂNICA DE ALTA RESOLUÇÃO
}

\author{
ANTARCTIC COASTAL POLYNYAS THROUGH HIGH RESOLUTION OCEANIC REANALYSIS
}

\author{
Camila Cristina HASHIMOTO, Mauricio Magalhães MATA \\ Universidade Federal do Rio Grande. Av. Itália, s/n - km 8, Carreiros, Rio Grande - RS. \\ Emails: cahashimoto@furg.br; mauricio.mata@furg.br

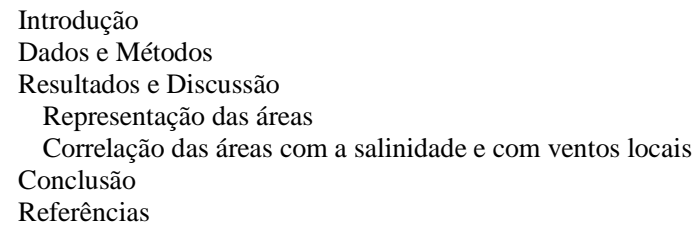

RESUMO - As polínias costeiras são conhecidas como “fábricas” de gelo marinho com importância, desde aspectos climáticos até biológicos. Os processos que ocorrem nas polínias afetam a formação de águas de fundo, devido ao efeito brine. A alternativa mais usada para investigar as polínias é através do sensoriamento remoto, com o avanço tecnológico em assimilação de dados em modelos oceânicos, os produtos de reanálises oceânicas passam a ser uma alternativa. Assim, este estudo comparou a representação das áreas das polínias no produto da GLO-HR com as observações satelitais a partir da concentração de gelo adquirida pelo sensor AMSR-E. A média da área diária de onze polínias costeiras foi calculada no período de congelamento (2013 - 2016) e a representação foi condizente com as posições relatadas, pois a resolução mais refinada da GLO-HR retrata áreas com maior precisão, porém menores. A salinidade aumentou em quase todas as polínias, porém não houve correlação com a área. A correlação com os ventos demonstrou a direção determinante nas fases de abertura e fechamento das polínias. Concluindo, há mais fatores que auxiliam no fechamento do que na abertura das áreas, e compreender a variabilidade das polínias permite detalhamento nos modelos oceânicos, principalmente aos processos ocorridos na plataforma continental.

Palavras-chave: polínias costeiras, Antártica, reanálise de alta resolução, gelo marinho.

\begin{abstract}
Coastal polynyas are known as sea ice factories with great importance from climatic to biological aspects. The processes that occur in the polynyas affect the primary productivity and the formation of bottom water due to brine effect. Despite the Polynyas were mainly investigated using remote sensing, the ocean reanalysis product are becoming an important tool to these studies. This study compared the eleven polynyas areas representation in the reanalysis products and the observations of satellites from sea ice concentration data (by the AMSR-E sensor). The daily average areas of the eleven Antarctic's coastal polynyas were calculated in the freezing period (2013 - 2016). The representation of the areas was consistent with previously reported by the literature. It is possible that the most improved resolution portrays accurate and minor areas. Salinity increased in almost all polynyas throughout their development, but there was no correlation with the associated areas. There was significant correlation with winds direction that favors the sea ice divergence, and hence the sea ice formation to each polynya. Finally, understanding their variability allows the refinement of the models by detailing in such areas, mostly as regards in the processes into continental shelf.
\end{abstract}

Keywords: coastal polynyas, Antarctic, reanalysis product, sea ice.

\section{INTRODUÇÃO}

A formação de Água de Fundo Antártica (AABW - Antarctic Bottom Water), em síntese, envolve a mistura de águas densas originadas do processo de interação com a atmosfera e gelo marinho que ocorre na camada superficial.

A massa de água mais densa, afunda na plataforma continental e escoa pelo talude, misturando-se com massas de água de profundidade intermediária até alcançar o assoalho oceânico (Ohshima et al., 2003; Jacobs, 2004). O processo de formação da AABW é conhecido (Orsi et al.,, 1999; Williams et al.,, 2010; Talley, 2013), bem como as áreas onde este processo ocorre como, por exemplo, o Mar de Weddel (Fooldvik et al.,, 2004), Mar de Ross (Gordon et al., 2009) e a região da Terra de Adélie, que compreende a Polínia de Mertz (MP - Mertz Polynya) (Williams et al., 2010). Recentemente, outras regiões como a Polínia de Cape Darnley (CDP Cape Darnley Polynya) (Ohshima et al., 2013) e a Polínia da Baía de Vincennes (VBP Vincennes Bay Polynya) (Kitade et al., 2014) foram reconhecidas como áreas de formação de AABW (Figura 1), indicando que ainda há lacunas sobre o entendimento desse processo, principalmente, quando relacionados às polínias, impactando sua representação nos modelos climáticos. 


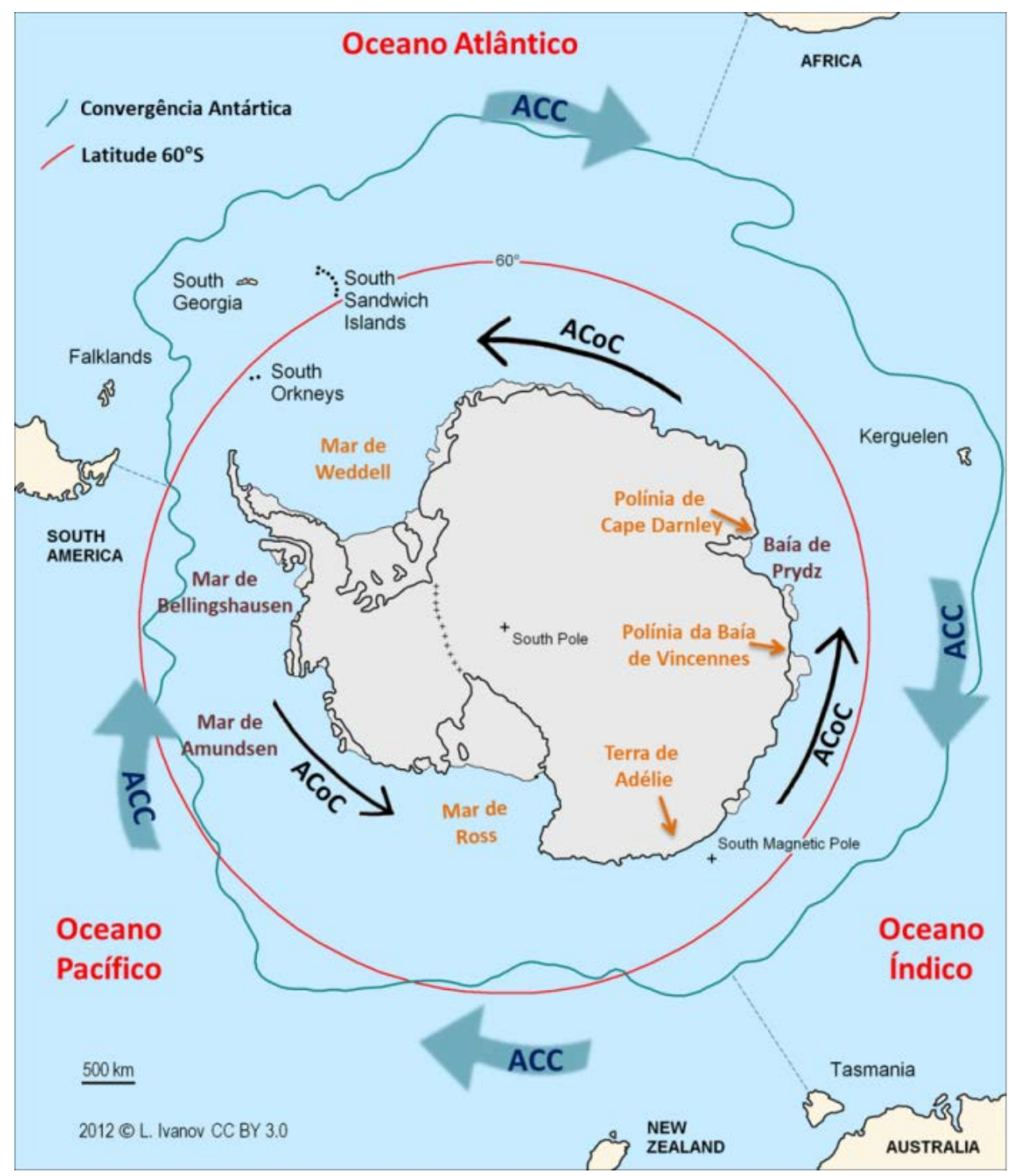

Figura 1 - Oceano Austral com limite dinâmico através da Convergência Antártica (linha azul) e limite definido pela latitude de $60^{\circ} \mathrm{S}$ (linha vermelha). Próximo ao continente, a Corrente Costeira da Antártica (ACoC) e afastada está a Corrente Circumpolar Antártica (ACC). Em vermelho estão representados os três principais oceanos Atlântico, Pacífico e Î́ndico; em laranja, as áreas de formação da AABW. Fonte: Adaptado de Geographic Names and Bulgarian Participation (Ivanov, L. and N. Ivanova, 2018).

A CDP tem alta taxa de produção de gelo marinho, capaz de produzir a variedade mais salina da Dense Shelf Water (DSW) (Ohshima et al., 2013), massa de água precursora de AABW. A região da VBP tem menor taxa de produção de gelo marinho em relação à CDP (Tamura et al., 2008), todavia produz uma variedade da DSW cuja contribuição nas camadas intermediárias é importante para a formação da AABW (Kitade et al., 2014). Atualmente, especula-se que outras polínias também têm capacidade de armazenar e exportar variações de DSW e, dessa forma, contribuir com a formação da AABW ( Williams et al., 2010; Ohshima et al., 2013). O volume da DSW formada nas polínias do leste antártico foi estimado em cerca de 7,58 Sv, onde $1 \mathrm{~Sv}=106$ m3.s-1, (Kusahara et al., 2010,
2011), portanto a contribuição individual de cada polínia pode ser pequena, mas a combinação de todas pode ser significante.

Nas últimas décadas, as propriedades das águas do oceano Austral estão apresentando mudanças. As tendências do gelo marinho diferem regionalmente. Nos Mares de Bellingshausen e Amundsen, a tendência é de forte diminuição na cobertura de gelo marinho (Zwally et al., 2002), diminuindo a permanência do gelo em 3,3 meses por década, entre 1979 à 2011 (Hobbs et al., 2016). Nas regiões dos mares de Weddell e Ross a tendência é positiva (Zwally et al., 2002), crescendo $1,7 \%$ por década entre 1979 e 2015 (Comiso et al., 2017). A extensão total do gelo marinho na Antártica está aumentando (Hobbs et al., 2016). Porém, recentemente, a extensão do gelo marinho 
registrou seu declínio mais acentuado com a menor extensão de gelo marinho desde 1979, possivelmente devido à sequência de fortes tempestades oriundas da baixa pressão sobre a região do Mar de Amundsen (Turner et al., 2017). A tendência de duração do gelo marinho reflete uma resposta combinada de vários componentes atmosféricos, porém o sistema não é linear (Turner et al., 2009; Matear et al., 2015; Comiso et al., 2017). A interação destes precisa ser melhor compreendida, pois cada setor responde de maneira distinta para cada modo de variabilidade climática (Hobbs et al., 2016).

Apesar da tendência de aumento ser registrada desde 1979 (Zwally et al., 2002; Turner et al., 2016; Comiso et al., 2017), a dificuldade de reprodução do gelo marinho nos modelos climáticos nos faz pensar que existe algum mecanismo faltando para simular precisamente o aumento da extensão de gelo (Williams et al., 2010), principalmente nos processos que ocorrem na plataforma continental como a formação do gelo marinho dentro das polínias costeiras.

As polínias costeiras são áreas não lineares no oceano, onde o gelo marinho é formado, podendo estar cobertas com gelo recémformado com a superfície mantida livre ou parcialmente livre de gelo (WMO, 1970; Smith et al., 1990). Além da importância na formação da DSW, as polínias têm um importante papel na manutenção da produtividade biológica, pois são os primeiros sistemas a ser expostos à radiação solar no início da primavera (Mundy \& Barber, 2001). O crescimento e acumulação da biomassa fitoplanctônica é maior dentro das polínias do que nas águas adjacentes ( $\mathrm{Li}$ et al., 2016), como resultado da alta produtividade primária, outros organismos de diferentes níveis tróficos são agregados nessas regiões (Arrigo, 2003; Malpress et al., 2017; Labrousse et al., 2018). As polínias também desempenham um papel nos ciclos biogeoquímicos, visto que a formação e exportação da DSW na região da MP é um mecanismo efetivo para transferir carbono antropogênico para o fundo do oceano (Shadwick et al., 2014).

As maiores polínias costeiras da Antártica foram mapeadas por Nihashi \& Ohshima (2015), através da sua frequência de ocorrência calculada a partir da concentração diária de gelo marinho (SIC - Sea Ice Concentration) estimada pelo algoritmo NT2 (NASA Team 2) (Nihashi \&
Ohshima, 2015). O NT2 utiliza os dados de Temperatura de Brilho, adquiridos pelo sensor AMSR-E (Advanced Microwave Scanning Radiometer for Earth Observing System) a bordo do satélite Aqua da NASA, para calcular a SIC (Markus \& Cavalieri, 2000). Os limites adotados para o cálculo das áreas são definidos por SIC $<30 \% r$, seguindo os estudos de Nihashi et al., (2009) e Iwamoto et al., (2014).

As polínias costeiras são moldadas através do balanço entre a resistência de arrasto do gelo marinho e a força para empurrá-lo para fora da costa (Maqueda, 2004). À medida que o vento empurra o gelo recém formado para longe da costa, a água do mar forma mais cristais de gelo, mantendo a área da polínia livre de gelo marinho ou “aberta”. Quando o vento não é intenso o suficiente para arrastar as espículas de gelo, mas há perda de calor suficiente para congelar a superfície do mar, o gelo marinho formado encobre a área da polínia, "fechandoa”. Assim, a área da polínia pode aumentar ou diminuir várias vezes o seu tamanho durante o período de congelamento ou até mesmo durante um período de 24h (Ciappa \& Budillon, 2012). Finalmente, os processos que ocorrem nas polínias afetam a formação de águas de fundo, os fluxos de calor, sal, a variabilidade do gelo marinho, os processos biológicos e os ciclos biogeoquímicos. Contudo, as polínias não são bem representadas nos modelos climáticos de larga escala, limitando a capacidade de projetar mudanças físicas e biológicas relacionadas a elas (Arrigo, 2003; Tamura et al., 2008).

Logo, compreender a variabilidade destas áreas e aprimorar os modelos oceânicos para um maior detalhamento das polínias costeiras, torna-se pertinente, sobretudo quando as observações in situ são limitadas durante o verão e escassas no inverno.

Logo, a compreensão dos processos físicos que ocorrem na plataforma continental e envolvem as polínias costeiras é dificultada. As alternativas mais usadas para investigar a dinâmica das polínias são através de sensoriamento remoto, por meio de radiômetros de micro-ondas passivas como SSM/I (Special Sensor Microwave Imager) e AMSR-E, devido à transparência da atmosfera para esses comprimentos de onda e curto tempo de revisitação (duas a três vezes por dia); sensores termais na faixa do infravermelho como AVHRR (Advanced Very High Resolution 
Radiometer) e MODIS (Moderate Resolution Imaging Spectroradiometer) (Drucker, 2003; Ciappa et al., 2012; Ciappa \& Pietranera 2013; Paul et al., 2015) e às imagens SAR (Synthetic Aperture Radar) na banda X, pois apresentam resoluções espaciais mais finas (Parmiggiani, 2006; Hollands et al., 2013). Contudo, as lacunas temporais quando se utiliza dados de sensoriamento remoto, podem ser minimizadas quando se soma às simulações de modelos oceânicos. A partir dessa ideia, os produtos de reanálise oceânica são ferramentas indispensáveis para investigar os processos oceânicos.

A eficácia das reanálises está na síntese das informações fornecidas através de modelos oceânicos, fluxos atmosféricos e dados oceanográficos in situ, unidos por um método de assimilação (Cushman-Roisin \& Beckers, 2011). As informações fornecidas através das reanálises são amplamente utilizadas para prever respostas do oceano, como a variabilidade do nível do mar (Wunsch et al., 2007), distribuição e tendências da temperatura e salinidade na plataforma continental da Antártica (Schmidtko et al., 2014), análises multidecadais sobre o afundamento de dióxido de carbono no oceano Austral (Landschützer et al., 2015), variabilidade e troca do conteúdo de calor nos oceanos (Palmer et al., 2017), entre outros. A relativa alta resolução espacial e temporal das reanálises atuais permite captar processos que ocorrem em escalas espaciais e temporais muito próximas da dinâmica das polínias costeiras, principalmente durante 0 período de congelamento.

Neste cenário, este trabalho visa utilizar a reanálise oceânica de alta resolução "Global Analysis Forecast Physics 1/12" (GLO-HR) como fonte de dados, de modo a validar a representação das áreas das polínias costeiras, tendo como base a reprodução via sensoriamento remoto (Nihashi \& Ohshima, 2015). Além deste, correlacionar a variabilidade das áreas das polínias costeiras com a salinidade na coluna de água e com o padrão de ventos sobre as mesmas.

\section{DADOS E MÉTODOS}

O oceano Austral (Figura 1) está sob regime de duas principais correntes a Corrente Circumpolar Antártica (ACC - Antarctic Circumpolar Current), entre as latitudes de $45-60^{\circ} \mathrm{S}$, a qual conecta três grandes bacias oceânicas (oceanos Pacífico, Índico e Atlântico); e circundando a plataforma continental está a Corrente Costeira da Antártica (ACoC - Antarctic Coastal Current), impulsionada principalmente pelos Ventos de Leste, fluindo para oeste e paralela ao continente (Núñez-Riboni \& Fahrbach, 2009), influenciando assim o regime oceanográfico ao redor do continente antártico (Fahrbach et al., 1992). Próximo à margem continental, os Ventos de Leste (circumpolares) influenciam na extensão zonal das polínias (Massom et al., 1998), variando sua área de acordo com a sua intensidade, ao passo que, os Ventos Catabáticos contribuem para a formação, manutenção e exportação do gelo marinho.

A plataforma continental da Antártica não é uniforme e pode ser dividida em setores marcados por mares ou baías como Mar de Weddell, Mar de Bellingshausen, Mar de Amundsen, Mar de Ross e Baía de Prydz. Cada setor possui suas próprias características oceanográficas e climáticas (O’Brien et al., 2014), como os giros ciclônicos nos mares de Weddell e Ross; e na baía de Prydz
(Williams et al., 2016). A batimetria da plataforma continental influencia nos processos costeiros, auxiliando na intrusão de uma massa de água relativamente quente próximo a polínia de Bellingshausen (BeP) (Parent et al., 2015) ou, o relevo mais alto na plataforma próxima a polínia de Barrier (BaP) impede que parte da DSW formada pela polínia se misture com o giro ciclônico que ocorre na Baía de Prydz (Williams et al., 2016).

O produto de reanálise oceânica utilizado é a GLO-HR, a qual fornece previsões e estimativas sobre a circulação oceânica e parâmetros físicos dos oceanos. Para tal, dois modelos numéricos, relativos ao oceano e ao gelo marinho, são acoplados com uma forçante atmosférica. Sendo assim, GLO-HR conecta o modelo oceânico NEMO (Nucleus for European Modelling of the Ocean) (Madec \& Imbard, 1996) com o modelo termodinâmico de gelo marinho LIM2 (Louvainla-Neuve-Sea Ice Model) (Timmermann et al., 2005), cuja forçante atmosférica é integrada através dos dados do ECMWF (European Centre for Medium-Range Weather Forecast) de modo que é originado um conjunto de parâmetros físicos globais que descrevem a história da atmosfera e dos oceanos.

O produto da reanálise GLO-HR tem alta 
resolução espacial $\left(1 / 12^{\circ}\right.$ latitude $\mathrm{x} \quad 1 / 12^{\circ}$ longitude), aproximadamente $2-3 \mathrm{~km}$ em altas latitudes (Madec \& Imbard, 1996), o que pode tornar possível a compreensão de processos físicos que ocorrem em menores escalas, como as polínias. A vantagem é a cobertura temporal que compreende todo o inverno; neste trabalho foram utilizados os parâmetros de SIC, que expressa a porcentagem da cobertura de gelo dentro de cada célula da GLO-HR, e salinidade na profundidade de 300m na área das polínias. O período de congelamento considerado foi de maio a setembro, dos anos de 2013 até 2016. No entanto, no hemisfério Sul, a cobertura espacial compreende até $77^{\circ} \mathrm{S}$ de latitude, logo a Polínia de Ross não está inclusa na sua totalidade, pois ela começa além de $78^{\circ} \mathrm{S}$.

Para complementar as análises parâmetros de vento do produto de reanálise atmosférica ERA Interim como velocidade zonal $(u)$ e velocidade meridional $(v)$ à $10 \mathrm{~m}$ da superfície foram utilizados. ERA - Interim apresenta o ciclo hidrológico combinado com a circulação estratosférica (Berrisford et al., 2011), a qual combina três componentes: atmosfera, superfície terrestre e ondas no oceano com dados de sensoriamento remoto e in situ (Dee et al., 2011). A assimilação dos dados é feita em ciclos de 12h, a cada ciclo as observações disponíveis são combinadas com as observações anteriores para estimar a evolução atmosférica global e da superfície abaixo (Dee et al., 2011). A resolução horizontal é de $0,75^{\circ}$ latitude x $0,75^{\circ}$ longitude e 60 níveis verticais entre a superfície até a pressão de 0,1hPa (aproximadamente $70 \mathrm{~km}$ ).

As áreas das polínias costeiras foram definidas como $\mathrm{SIC} \leq 30 \%$ e que estavam próximas às margens continentais. Tal definição é baseada nas observações por satélite (Nihashi \& Ohshima, 2015), provenientes de dados adquiridos a partir

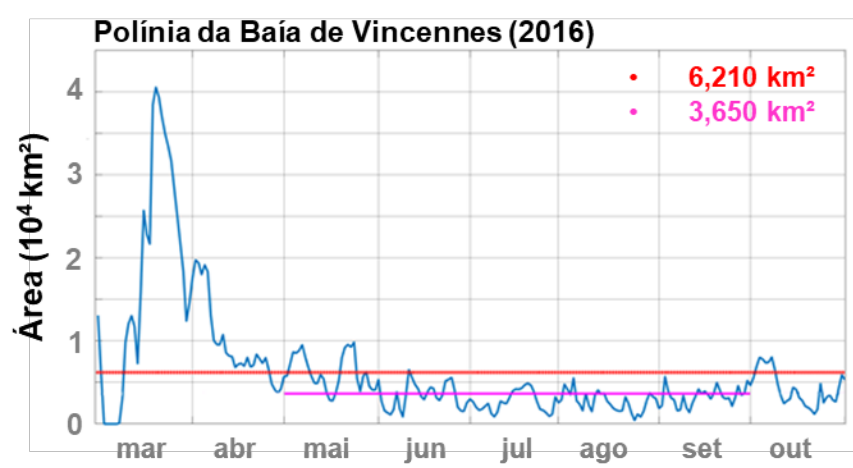

de sensores micro-ondas (AMSR-E) e infravermelho termal (MODIS), os quais utilizam o algoritmo NT2, para calcular a SIC e estimar a espessura do gelo marinho (Nihashi et al., 2009; Iwamoto et al., 2014). Estas áreas foram calculadas diariamente e depois mediadas durante o período de congelamento considerado de maio a setembro, menor em relação ao trabalho de Nihashi \& Ohshima (2015). Dessa forma, o grau de incerteza das médias das áreas é diminuído, pois não ocorre interferência do descongelamento durante o fim do verão e na primavera (Figura 2). Em seguida, foi calculada a frequência de ocorrência de cada célula da GLO-HR que SIC $\leq$ $30 \%$, assim a área recorrente das polínias foi estabelecida. Ambos os cálculos foram realizados com o intuito de comparar com as áreas obtidas através do sensoriamento remoto realizado por Nihashi \& Ohshima (2015), a fim de avaliar a representatividade das mesmas áreas na GLO-HR.

A variabilidade diária de cada polínia foi considerada por meio do Coeficiente de Variação (CV) calculado através do desvio padrão diário (DP) em relação à média do período de congelamento (para cada polínia individualmente):

$$
C V=\frac{D P}{\text { Média }} \quad \text { Eq. } 1
$$

A influência dos ventos locais interfere nas fases de abertura e fechamento das polínias costeiras, as médias diárias das componentes de $u$ e $v$ foram calculadas. Posteriormente, foi realizada a correlação linear entre as componentes do vento e a variabilidade diária de cada área, a fim de avaliar a importância desta nas fases de abertura e fechamento, identificando qual a direção que favorece a divergência do gelo marinho (portanto, mantém a polínia aberta).

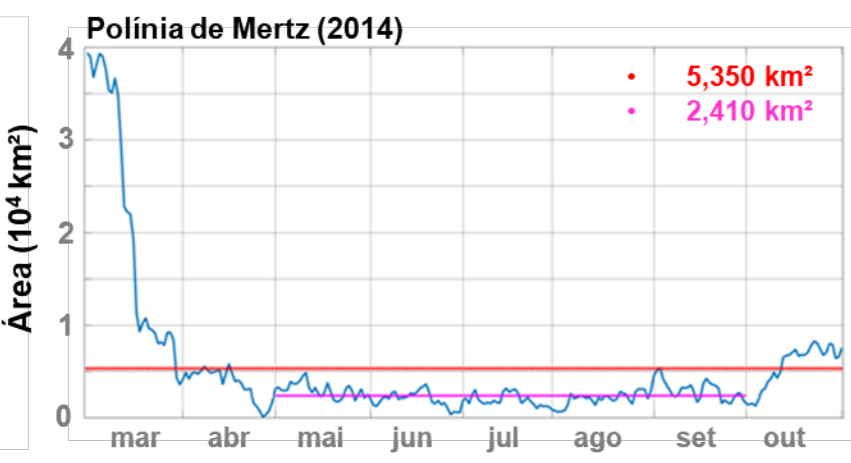

Figura 2 - Série temporal das áreas diárias das polínias da Baía de Vincennes (acima) e de Mertz (abaixo). As médias do período de congelamento de março a outubro (vermelho) e maio a setembro (rosa) para ambas. 


\section{RESULTADOS E DISCUSSÃO}

\section{Representação das áreas}

As polínias costeiras representadas no produto da GLO-HR (Figura 3) coincidem com as posições nas observações por satélite, sendo reproduzidas de acordo com o observado e posicionadas adjacente às feições como fast ice e glacier tongues.

As médias anuais das áreas calculadas estão na Tabela 1 com seus respectivos DP e CV.
Todas apresentaram médias menores, como esperado, quando comparadas com as médias do período de congelamento das observações por satélite.

A principal razão é a diferença no período de congelamento usado, cuja diferença é de 3 meses, sendo 5 meses (maio a setembro) na GLO-HR e nas observações por satélite 8 meses (março a outubro).
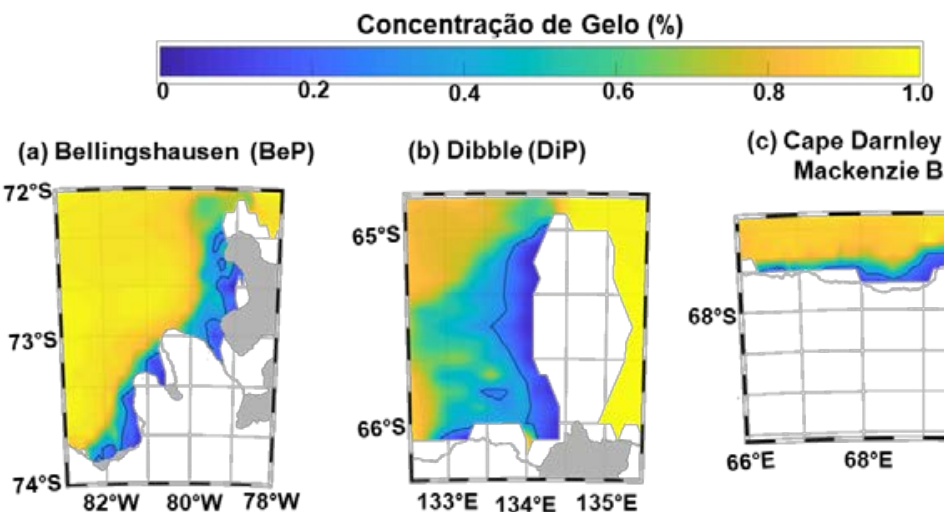

(b) Dibble (DiP)

(c) Cape Darnley (CDB) e
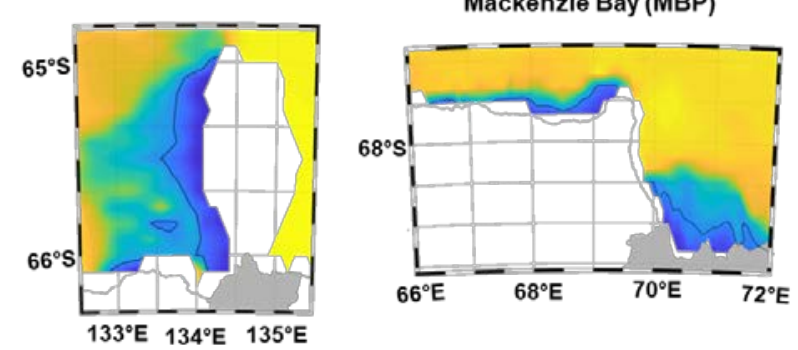

(d) Ronne (RONP)

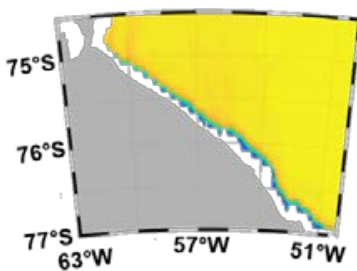

(e) Mertz (MP)
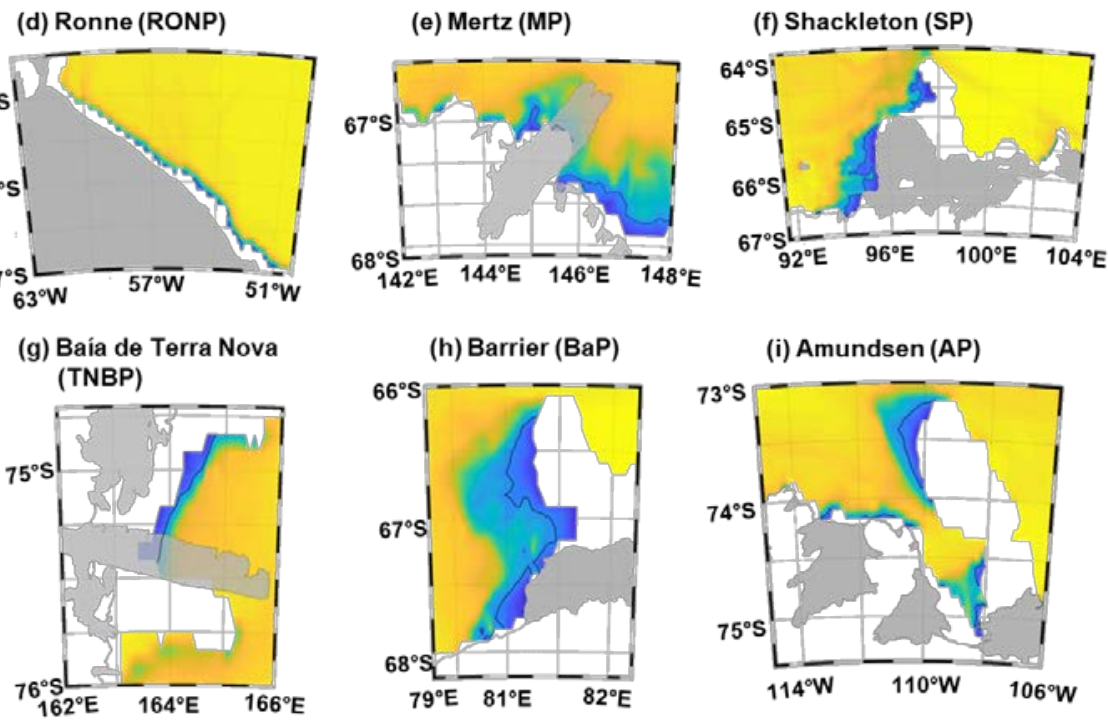

(h) Barrier (BaP)

(i) Amundsen (AP)
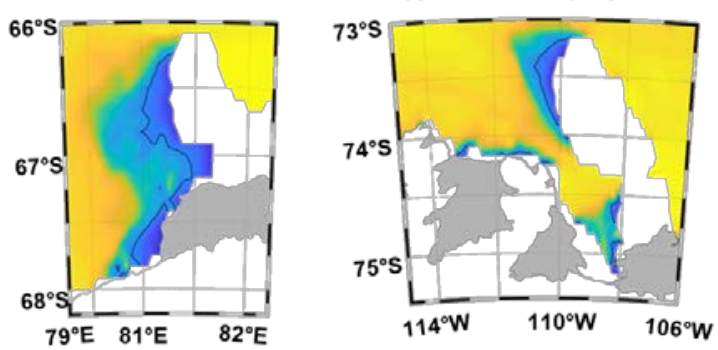

(j) Dalton (DaP)

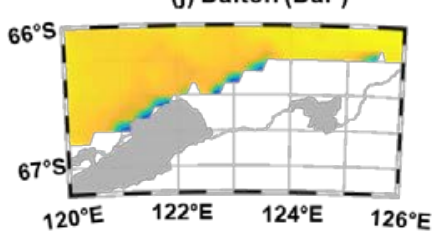

(k) Baia de Vincennes (VBP)

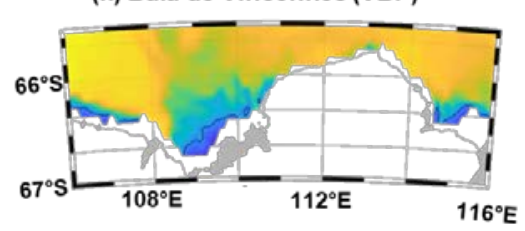

Figura 3 - Concentração do gelo marinho reproduzida no produto de reanálise GLO-HR. A linha azul representa o limite de SIC $\leq 30 \%$ nas polínias costeiras, calculadas durante o período de congelamento (maio-setembro) de 2013 2016.

Além disto, foi observado que as polínias costeiras podem permanecer até 17 dias seguidos com a área fechada e, em seguida (4 a 7 dias), alcançar os máximos valores de extensão. Dessa maneira, os picos máximos das áreas são mantidos em menos tempo em relação à quantidade de dias que as mesmas permanecem fechadas, resultando em altos desvios padrões. Neste contexto, o CV das polínias estudadas na GLO-HR também é alto (Tabela 1), confirmando que todas têm bruscas alterações no tamanho das áreas, durante os quatro anos estudados, e tais alterações são frequentes, consideradas característica das polínias costeiras da Antártica. 
Tabela 1 - Média anual das áreas das polínias costeiras, calculadas através do produto da reanálise GLO-HR com seus respectivos desvios padrões (DP) e coeficientes de variação (CV), durante o período de congelamento (maio setembro) dos anos de 2013 - 2016. Na última coluna à direita, a área média do período de congelamento (março outubro) dos anos de 2003 - 2011, calculada através das observações por satélite em Nihashi e Ohshima (2015).

\begin{tabular}{c|c|c|c|c|c|c|c|c|c}
\hline \multicolumn{7}{c}{ Área $\mathbf{1 0}^{\mathbf{3}} \mathbf{k m}^{\mathbf{2}}$} \\
\hline Nome da Polínia & $\mathbf{2 0 1 3}$ & $\mathbf{C V}$ & $\mathbf{2 0 1 4}$ & $\mathbf{C V}$ & $\mathbf{2 0 1 5}$ & $\mathbf{C V}$ & $\mathbf{2 0 1 6}$ & $\mathbf{C V}$ & $\begin{array}{c}\text { Obs. } \\
\text { Satélite }\end{array}$ \\
\hline Cape Darnley (CDP) & $0,75 \pm 0,6$ & 0,8 & $1,20 \pm 0,8$ & 0,7 & $0,86 \pm 0,6$ & 0,7 & $1,06 \pm 0,6$ & 0,6 & $10,3 \pm 3,7$ \\
\hline $\begin{array}{c}\text { Baía de Mackenzie } \\
\text { (MBP) }\end{array}$ & $1,61 \pm 0,9$ & 0,6 & $1,53 \pm 0,9$ & 0,6 & $1,80 \pm 0.6$ & 0,3 & $1,90 \pm 0.8$ & 0,4 & $3,9 \pm 2,1$ \\
\hline Barrier (BaP) & $4,21 \pm 2,7$ & 0,6 & $4,29 \pm 2,9$ & 0,6 & $3,39 \pm 2,7$ & 0,8 & $2,82 \pm 2,1$ & 0,7 & $6,0 \pm 2,7$ \\
\hline Shackleton (SP) & $6,99 \pm 4,5$ & 0,6 & $6,43 \pm 3,3$ & 0,5 & $5,13 \pm 2,9$ & 0,5 & $6,39 \pm 3,1$ & 0,4 & $7,5 \pm 3,6$ \\
\hline $\begin{array}{c}\text { Baía de Vincennes } \\
\text { (VBP) }\end{array}$ & $2,18 \pm 1,3$ & 0,6 & $2,68 \pm 1,1$ & 0,4 & $2,36 \pm 1,3$ & 0,5 & $3,65 \pm 1,9$ & 0,5 & $6,3 \pm 2,2$ \\
\hline Dalton (DaP) & $0,19 \pm 0,3$ & 1,5 & $0,37 \pm 0,5$ & 1,4 & $0,34 \pm 0,4$ & 1,3 & $0,92 \pm 1,0$ & 1,0 & $3,7 \pm 2,0$ \\
\hline Dibble (DiP) & $2,57 \pm 1,4$ & 0,5 & $2,38 \pm 1,1$ & 0,5 & $1,96 \pm 1,0$ & 0,5 & $2,81 \pm 1,6$ & 0,6 & $2,4 \pm 0,27$ \\
\hline Mertz (MP) & $2,42 \pm 1,0$ & 0,4 & $2,41 \pm 0,9$ & 0,3 & $2,63 \pm 0,9$ & 0,3 & $2,65 \pm 0,9$ & 0,3 & $9,7 \pm 4,4$ \\
\hline $\begin{array}{c}\text { Baía de Terra Nova } \\
\text { (TNBP) }\end{array}$ & $0,61 \pm 0,5$ & 0,9 & $0,53 \pm 0,4$ & 0,8 & $0,91 \pm 0,6$ & 0,6 & $0,93 \pm 0,9$ & 1,0 & $3,6 \pm 2,1$ \\
\hline Amundsen (AP) & $2,88 \pm 1,9$ & 0,6 & $1,59 \pm 1,7$ & 1,1 & $1,72 \pm 1,2$ & 0,7 & $3,49 \pm 1,9$ & 0,5 & $7,7 \pm 3,6$ \\
\hline Bellingshausen (BeP) & $3,90 \pm 6,8$ & 1,7 & $4,61 \pm 7,8$ & 1,6 & $3,61 \pm 5,4$ & 1,5 & $4,22 \pm 3,8$ & 0,9 & $4,9 \pm 2,8$ \\
\hline $\begin{array}{c}\text { Plataforma de Gelo } \\
\text { Ronne (RONP) }\end{array}$ & $1,05 \pm 1,5$ & 1,4 & $1,37 \pm 0,2$ & 1,5 & $1,07 \pm 1,1$ & 1,1 & $2,70 \pm 2,0$ & 0,7 & $2,3 \pm 2,7$ \\
\hline
\end{tabular}

O cálculo da frequência de ocorrência (Figura confrontado com as observações de Nihashi \& 4) fornece a posição regular de cada polínia Ohshima (2015), algumas representações de representada na GLO-HR. No entanto, quando fast ice não foram condizentes com o esperado

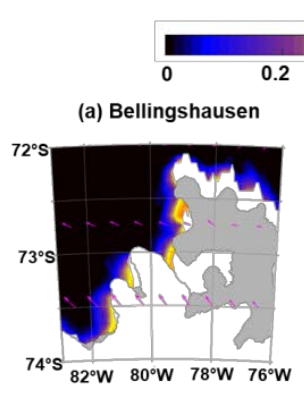

Frequência de Ocorrência (\%)

(d) Ronne
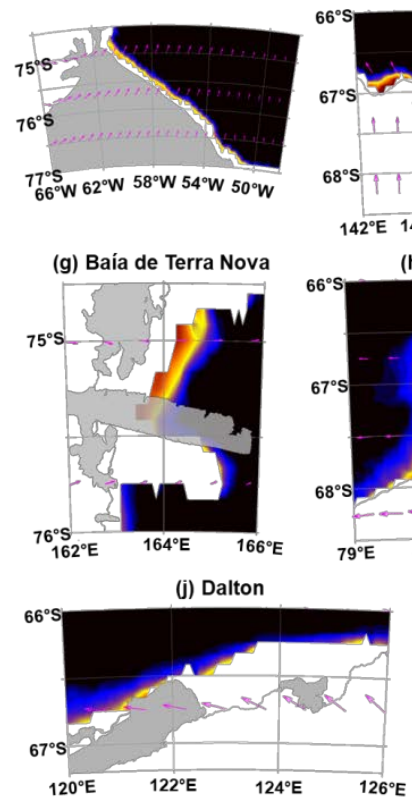

(b) Dibble

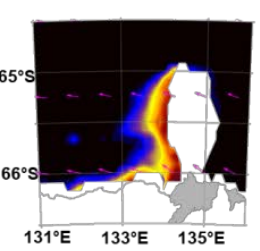

(e) Mertz

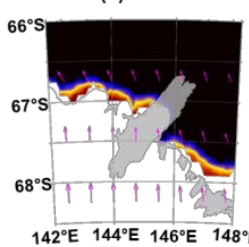

(h) Barrier

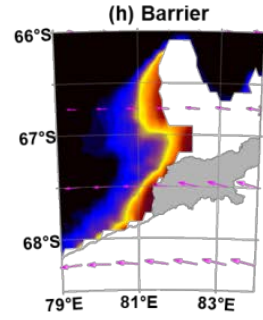

(c) Cape Darnley e

Baia de Mackenzie

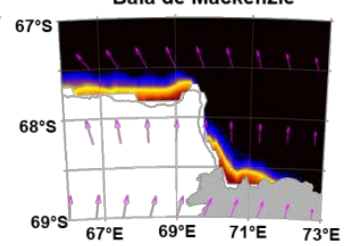

(f) Shackleton

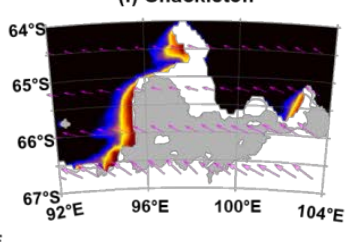

(i) Amundsen

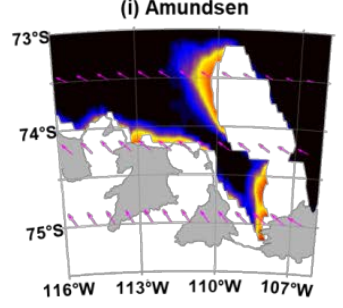

(k) Baía de Vincennes

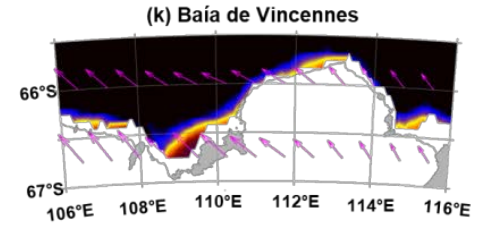

Figura 4 - Frequência de ocorrência calculada a partir do produto de reanálise GLO-HR do período de congelamento (maio a setembro) entre os anos de 2013 - 2016. As setas indicam a direção média do vento à 10m da superfície sob a área da polínia. 


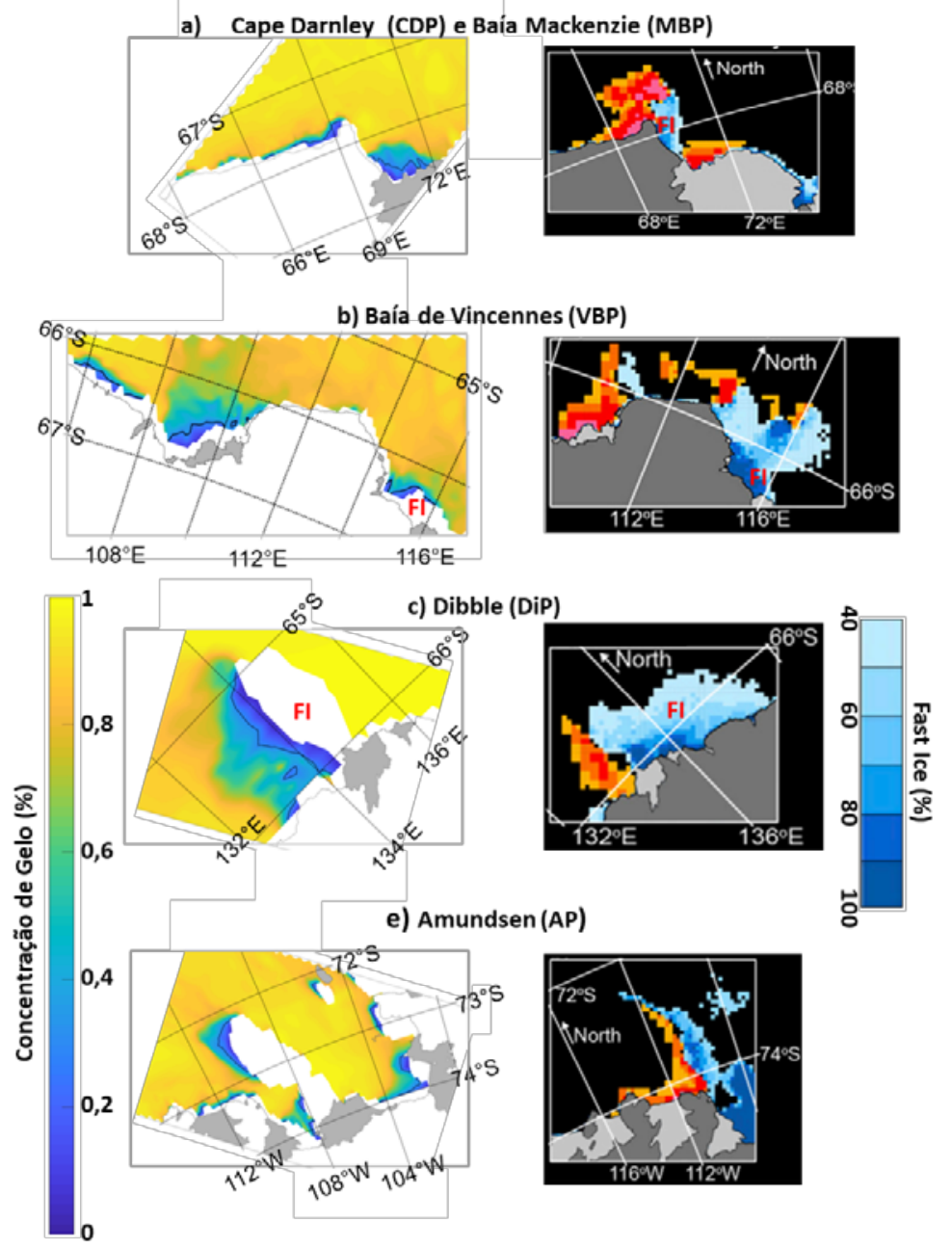

Figura 5 - Representação do fast ice (FI) através do produto da reanálise GLO-HR (esquerda) comparada com as observações por satélites à direita (adaptado de Nihashi e Ohshima, 2015).

(Figura 5). Apesar da resolução refinada, a representação dos fast ice não ocorreu na CDP e esta apresentou área cerca de 10 vezes menor do que o observado. A ocorrência do fast ice nas polínias de VBP e na Polínia de Dibble (DiP Dibble Polynya) não foi semelhante com a representação na GLO-HR. As polínias cujo fast ice não foi representado adequadamente tiveram a média das áreas significativamente menor em relação às descritas em Nihashi \& Ohshima (2015). Assim, a representação destas áreas pode estar comprometida, visto que o fast ice é importante na evolução da área (Maqueda, 2004).

Apesar das polínias de DaP, Polínia de Amundsen (AP - Amundsen Polynya) e a polínia da Baía de Terra Nova (TNBP - Terra Nova Bay Polynya) estarem bem representadas quanto aos seus fast ice, $a$ média das áreas são significativamente menores, estando entre as maiores diferenças entre as áreas calculadas na GLO-HR e através sensoriamento remoto. A MP apresentou média significativamente menor (aproximadamente, 4 vezes menor), o motivo é atribuído ao colapso do Mertz Glacier Tongue (MGT) em 2010 (Dragon et al., 2014).

Contudo, polínias como BaP, BeP, polínia de Shackleton (SP) e polínia da Plataforma de Gelo Ronne (RONP) foram bem representadas em relação ao tamanho das áreas, sendo que algumas polínias pode-se notar uma tendência de aumento na área, em relação aos 4 anos estudados, como em VBP, DaP, MP e RONP. A única polínia que apresenta redução da área ao longo dos anos é BaP.

\section{Correlação das áreas com a salinidade e com ventos locais}

A média da salinidade ao longo do período de congelamento na profundidade de $300 \mathrm{~m}$ aumentou em todas as polínias em pelo menos um dos anos estudados (Figura 6), porém não houve correlação com as áreas. Durante o período de congelamento, a TNBP e BeP, apresentaram a salinidade mais alta do lado oeste do continente antártico, fortificando o aumento da produção de gelo marinho localizada no Mar de Ross (Zwally, 2002; Comiso et al., 2017). Ainda, TNBP, BeP, MP, CDP, DiP e a polínia da Baía de Mackenzie 

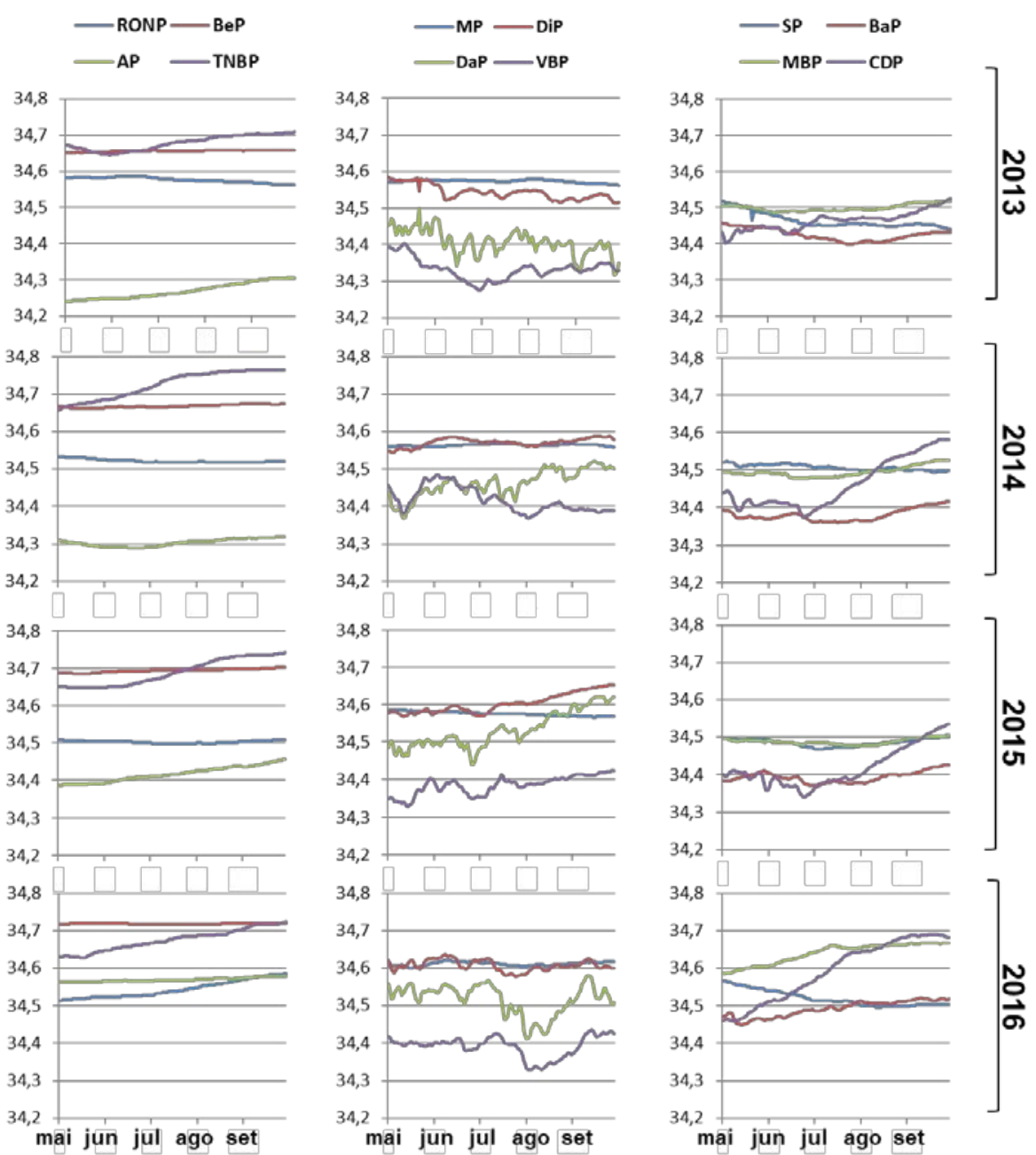

Figura 6 - Salinidade ao longo do período de congelamento dos anos de 2013 a 2016, na profundidade de 300m.

(MBP - Mackenzie Bay Polynya) alcançaram salinidade $>34,6$, valor em que a DSW é capaz de afundar e contribuir com a formação da AABW, tanto na Baía de Prydz quanto no Mar de Ross (Ciappa \& Pietranera 2013; Ohshima et al., 2013; Comiso et al., 2017).Os valores mais baixos de salinidade foram encontrados em AP, DaP e BaP. A salinidade baixa em AP e DaP já foram relatadas em estudos como Silvano et al. (2018) e (Parent et al., 2015), ambos sugerem que a injeção de sal relacionada com a formação do gelo marinho é suprimida pelo derretimento basal nas regiões. Já em $\mathrm{BaP}$, a baixa salinidade pode ser atribuída pela intrusão de massas de água menos salinas que em observações in situ apresentaram valores entre 34,4 e 34,5 (Williams et al., 2016). As correlações das componentes $u$ e $v$ com as respectivas áreas foram mais fortes com uma ou outra componente e estão apresentadas na tabela 2.

$O$ valor de $u$ e $v$ pode ser positivo ou negativo, cujo sinal indica apenas a direção do vento:

- $\quad$ u negativo, são ventos de quadrante Leste;
- $\quad$ u positivo, são ventos de quadrante Oeste;

- $v$ negativo, são ventos de quadrante Norte;

- $v$ positivo, são ventos de quadrante Sul;

Dessa maneira, as correlações negativas referem-se às áreas que aumentam com ventos Norte e/ou Leste e as correlações positivas fazem referência às áreas que aumentam com ventos Sul e/ou Oeste.

As áreas das polínias apresentaram melhor correlação com a direção que promove a divergência do gelo marinho entre o oceano e a feição adjacente (fast ice, glacier ou margem costeira). As exceções são CDP e MBP, localizadas na Baía de Prydz, nestes casos a correlação se dá com $u$ e $v$ (Tabela 2).

As direções que favorecem a divergência do gelo nos produtos da GLO-HR corroboram com àquelas encontradas nas observações por satélite na maioria das polínias (Tabela 3). As diferenças ocorreram em quatro polínias: SP, MP, AP e DaP e são mínimas, já que todas as direções se encontram no mesmo quadrante e as polínias DaP e MP apresentarem pouca correlação com $u$ ou $v$. 
Tabela 2 - Correlação da área diária com as componentes $u$ e $v$, ambas a $10 \mathrm{~m}$ da superfície. Em negrito estão as correlações onde $r>0,50$.

\begin{tabular}{l|c|c|c|c|c|c|c|c}
\hline \multicolumn{1}{c}{ Correlação Vento x Área } \\
\hline \multirow{2}{*}{ Nome da Polínia } & \multicolumn{3}{c}{ Componente Zonal (u) } & \multicolumn{3}{c}{ Componente Meridional $(v)$} \\
\cline { 2 - 9 } & $\mathbf{2 0 1 3}$ & $\mathbf{2 0 1 4}$ & $\mathbf{2 0 1 5}$ & $\mathbf{2 0 1 6}$ & $\mathbf{2 0 1 3}$ & $\mathbf{2 0 1 4}$ & $\mathbf{2 0 1 5}$ & $\mathbf{2 0 1 6}$ \\
\hline Cape Darnley (CDP) & $-0,50$ & $-0,59$ & $-0,40$ & $-0,54$ & 0,67 & 0,53 & 0,68 & 0,71 \\
\hline Baía de Mackenzie (MBP) & 0,59 & 0,65 & 0,60 & 0,37 & 0,59 & 0,57 & 0,57 & 0,58 \\
\hline Barrier (BaP) & $-0,50$ & $-0,63$ & $-0,51$ & $-0,79$ & $-0,26$ & $-0,25$ & $-0,02$ & $-0,27$ \\
\hline Shackleton (SP) & $-0,54$ & $-0,70$ & $-0,50$ & $-0,60$ & $-0,07$ & 0,06 & 0,02 & 0,13 \\
\hline Baía de Vincennes (VBP) & $-0,13$ & $-0,30$ & $-0,13$ & $-0,31$ & 0,53 & 0,64 & 0,76 & 0,45 \\
\hline Dalton (DaP) & 0,00 & $-0,03$ & 0,05 & $-0,28$ & 0,44 & 0,59 & 0,58 & 0,46 \\
\hline Dibble (DiP) & $-0,65$ & $-0,64$ & $-0,64$ & $-0,67$ & 0,18 & 0,09 & $-0,02$ & $-0,03$ \\
\hline Mertz (MP) & 0,51 & 0,32 & 0,36 & 0,36 & 0,46 & 0,47 & 0,55 & 0,39 \\
\hline Baía de Terra Nova (TNBP) & 0,69 & 0,63 & 0,67 & 0,79 & $-0,21$ & $-0,33$ & $-0,28$ & 0,05 \\
\hline Amundsen(AP) & $-0,48$ & $-0,31$ & $-0,20$ & $-0,21$ & 0,56 & 0,57 & 0,57 & 0,72 \\
\hline Bellingshausen (BeP) & $-0,30$ & $-0,55$ & $-0,34$ & $-0,44$ & 0,53 & 0,49 & 0,61 & 0,53 \\
\hline Plataforma de Gelo Ronne (RONP) & 0,60 & 0,57 & 0,67 & 0,77 & 0,32 & 0,52 & 0,33 & 0,51 \\
\hline
\end{tabular}

Tabela 3 - As direções que melhor favorecem o aumento das áreas representadas em GLO-HR (direita) e nas observações por satélite (esquerda) (Nihashi e Ohshima 2015); Foi adotado o Norte como $0^{\circ}$ e perpendicular à costa onde a polínia se localiza.

\begin{tabular}{c|c|c|c|c}
\hline \multirow{2}{*}{} & \multicolumn{4}{|c}{ Direção dos ventos } \\
\cline { 2 - 5 } & Obs. Satélite & \multicolumn{2}{c}{ GLO-HR } \\
\hline CDP & $151^{\circ}$ & $S S E$ & $141-163^{\circ}$ & $S E / S S E$ \\
\hline MBP & $212^{\circ}$ & $S S O$ & $183-206^{\circ}$ & $S / S S O$ \\
\hline BaP & $95^{\circ}$ & $E$ & $88-100^{\circ}$ & $E$ \\
\hline SP & $133^{\circ}$ & $S E$ & $92-116^{\circ}$ & $E / E S E$ \\
\hline VBP & $128^{\circ}$ & $S E$ & $122-143^{\circ}$ & $E S E / S E$ \\
\hline DaP & $108^{\circ}$ & $E S E$ & $134-173^{\circ}$ & $S E / S S E / S$ \\
\hline DiP & $113^{\circ}$ & $E S E$ & $102-116^{\circ}$ & $E S E$ \\
\hline MP & $158^{\circ}$ & $S S E$ & $169-203^{\circ}$ & $S / S S O$ \\
\hline TNBP & $276^{\circ}$ & $O$ & $260-281^{\circ}$ & $O$ \\
\hline AP & $119^{\circ}$ & $E S E$ & $129-175^{\circ}$ & $S E / S S E / S$ \\
\hline BeP & $113^{\circ}$ & $E S E$ & $97-164^{\circ}$ & $E / E S E / S E / S$ \\
\hline RONP & $249^{\circ}$ & OSO & $243-256^{\circ}$ & OSO \\
\hline
\end{tabular}

\section{CONCLUSÕES}

As áreas representadas no produto da GLOHR foram menores em relação às observações por satélite, como esperado, devido, principalmente, à diferença no período de congelamento. A má representação do fast ice em algumas polínias (CDP, VBP, DiP e AP) pode ter contribuído para a redução das áreas. Contudo, a resolução espacial da GLO-HR é mais refinada, logo é possível que as áreas das polínias sejam retratadas com maior precisão, pois são representadas no período de congelamento mais adequado, não tendo interferência do descongelamento do fim do verão ou da primavera.

O estudo das áreas das polínias através do sensoriamento remoto com alta resolução espacial (sensores na faixa do infravermelho e imagens SAR) apresenta limitações na presença de chuvas e nuvens, o que não ocorre quando se utiliza sensores de micro-ondas passivas, porém a resolução é inferior e o algoritmo usado (NT2) tende a subestimar a SIC (Nihashi et al., 2009), o que pode resultar em áreas aparentemente maiores. Por fim, é sugerido que as resoluções 
mais refinadas encontrem áreas menores quando comparadas aos dados de micro-ondas passivas, que por sua vez podem superestimar as áreas.

As feições adjacentes às polínias são importantes e contribuem com a evolução das áreas, devido ao efeito bloqueador do gelo marinho a barlavento e desenvolvimento da polínia a sotavento. Logo, a representação inadequada destas influenciam no cálculo das áreas, como ocorreu com a CDP, onde o CDIB não foi representado na GLO-HR, diminuindo a média da área representada. Desse modo, é possível observar que polínias com feições adjacentes são suscetíveis ao arranjo dos ventos e ao agente bloqueador, sendo assim, cada área é condicionada às características locais como processos termodinâmicos e advectivos.

Não houve correlação da salinidade em 300m de profundidade com a variabilidade das áreas, pois a variabilidade da área é muito grande para correlacionarmos linearmente com a variabi- lidade da salinidade. Pois, o sistema que envolve a formação de gelo marinho e aumento da salinidade não foi explicado na GLO-HR.

Por outro lado, ao correlacionar a área diária com $u$ e $v$, notou-se que em todas as polínias a direção do vento é determinante nas fases de abertura e fechamento da polínia. Quando comparado com as observações por satélite, quatro polínias (DaP, MP, AP e SP) apresentaram pequenas diferenças nas direções que melhor divergem o vento, no entanto a direção que melhor se correlaciona com a fase de abertura das áreas ainda é proveniente do mesmo quadrante.

A redução da área ocorreu quando a direção do vento favoreceu a convergência do gelo marinho em direção à costa, fast ice ou do glacier adjacente. Assim, foi observado que há mais fatores que auxiliam no fechamento das áreas (convergência do gelo) do que na abertura das áreas (divergência do gelo).

\section{AGRADECIMENTOS}

Este estudo é uma contribuição para o INCT da Criosfera (CNPq 465680v2014-3 CAPES, 88887.13.6384/2017-00). CCH e MMM agradecem o apoio recebido da CAPES (bolsa de mestrado) e CNPq (306896/2015-0), respectivamente.

\section{REFERÊNCIAS}

ARRIGO, K.R. Phytoplankton dynamics within 37 Antarctic coastal polynya systems. Journal of Geophysical Research, v. 108, n. C8, p. 3271, 2003.

BEITSCH, A.; KERN, S.; KALESCHKE, L. Comparison of SSM/I and AMSR-E sea ice concentrations with ASPeCt ship observations around antarctica. IEEE Transactions on Geoscience and Remote Sensing, v. 53, n. 4, p. 1985-1996, 2015.

BERRISFORD, P.E. C. Atmospheric conservation properties in ERA-Interim. Quarterly Journal of the Royal Meteorological Society, v. Jully B, n. 137, p. 1381-1399, 2011.

CIAPPA, A. \& BUDILlON, G. The Terra Nova Bay (Antarctica) polynya observed by MODIS ice surface temperature imagery from May to June 2009. International Journal of Remote Sensing, v. 33, n. 14, p. 4567-4582, 2012.

CIAPPA, A. \& PIETRANERA, L. High resolution observations of the Terra Nova Bay polynya using COSMO-SkyMed XSAR and other satellite imagery. Journal of Marine Systems, v. 113-114, p. 42-51, 2013.

CIAPPA, A.; PIETRANERA, L.; BUDILLON, G. Observations of the Terra Nova Bay (Antarctica) polynya by MODIS ice surface temperature imagery from 2005 to 2010. Remote Sensing of Environment, v. 119, n. October, 2014, p. 158172, 2014.

COMISO, J.C.; GERSTEN, R.A.; STOCK, L.V.; JOHN TURNER, J.; PEREZ, G.J.; KOHEI, C.K. Positive Trend in the Antarctic Sea Ice Cover and Associated Changes in Surface Temperature. Journal of Climate, v. 30, n. 6, p. 2251-2267, 2017.

CUSHMAN-ROISIN, B. \& BECKERS, J.M. Data Assimilation. In: Introduction to Geophysical Fluid Dynamics: Physical and Numerical Aspects. Academic Press, p. 615-618, 2011.
DEE, D.P.; UPPALA, S.M.; SIMMONS, A.J.; BERRISFORD, P.; POLI, P.; KOBAYASHI, S.; ANDRAE, U.; BALMASEDA, M.A.; BALSAMO, G.; BAUER, P.; BECHTOLD, P.; BELJAARS, A.C.M.; VAN DE BERG, L.; BIDLOT, J.; BORMANN, N.; DELSOL, C.; DRAGANI, R.; FUENTES, M.; GEER, A.J.; HAIMBERGER, L.; HEAL, Y.S.B.; HERSBACH, H.; HÓLM, E.V.; ISAKSEN, L.; KÅLLBERG, P.; KÖHLER, M.; MATRICARDI, M.; MCNALLY, A.P.; MONGE -BANWMORCRETTE, J. J.; PARK, B.K.; PEUBEY, C.; ROSNAY, P.; TAVOLATO, C.; THÉPAUT, J.N.; VITART, F. The ERA-Interim reanalysis: Configuration and performance of the data assimilation system. Quarterly Journal of the Royal Meteorological Society, v. 137, n. 656, p. 553-597, 2011.

DRAGON, A.C.C.; MARIE-NOËLLE, H.; CHRISTOPHE H.; JEAN-BENOÎT, C. A note on the intraseasonal variability in an Antarctic polynia: Prior to and after the Mertz Glacier calving. Journal of Marine Systems, v. 130, p. 46-55, 2014.

DRUCKER, R. Observations of ice thickness and frazil ice in the St. Lawrence Island polynya from satellite imagery, upward looking sonar, and salinity/temperature moorings. Journal of Geophysical Research, v. 108, n. C5, p. 1-18, 2003.

FAHRBACH, E.; ROHARDT, G.; KRAUSE, G. The $\{$ A $\}$ ntarctic $\{C\}$ oastal $\{C\}$ urrent in the southeastern $\{W\}$ edell \{S\}ea. Polar Biology, v. 12, p. 171-182, 1992.

FOOLDVIK, A.; GAMMELSRØD, T.; ØSTERHUS, S.; FAHRBACH, E.; ROHARDT, G.; SCHRÖDER, M.; NICHOLLS, K.W.; PADMAN, L.; WOODGATE, R.A Ice shelf water overflow and bottom water formation in the southern Weddell Sea 2035. J. Geophys. Res, v. 109, n. C02015, p. C02015, 2004.

GORDON, A.L.; ORSI, A.; MUENCH, R.; VISBECK, M. Western Ross Sea continental slope gravity currents. Deep- 
Sea Research Part II: Topical Studies in Oceanography, v. 56, n. 13-14, p. 796-817, 2009.

HEIL, P. Atmospheric conditions and fast ice at Davis, East Antarctica: A case study. Journal of Geophysical Research: Oceans, v. 111, n. 5, p. 1-15, 2006.

HEIL, P.E GERLAND, S.E GRANSKOG, M.A. An Antarctic monitoring initiative for fast ice and comparison with the Arctic. The Cryosphere Discussions, v. 5, n. 5, p. 24372463, 2011.

HOBBS, W.R.; MASSOM, R.; STAMMERJOHN, S.; REID, P.; GUY WILLIAMS,'G.; MEIER'W. A review of recent changes in Southern Ocean sea ice, their drivers and forcings. Global and Planetary Change, v. 143, p. 228-250, 2016.

HOLLANDS, T.; V. HAID, V.; DIERKING, W.; TIMMERMANN, R.; EBNER, L. Sea ice motion and open water area at the Ronne Polynia, Antarctica: Synthetic aperture radar observations versus model results. Journal of Geophysical Research: Oceans, v. 118, n. 4, p. 1940-1954, 2013.

IWAMOTO, K.; OHSHIMA, K. I.; TAMURA, T. Improved mapping of sea ice production in the Arctic Ocean using AMSR-E thin ice thickness algorithm. Journal of Geophysical Research: Oceans, v. 119, p. 3574-3594, 2014.

JACOBS, S. S. Bottom water production and its links with the thermohaline circulation. Antarctic Science, v. 16, n. 4, p. 427-437, 2004.

KITADE, Y.; SHIMADA, K.; TAMURA, T.; WILLIAMS, G. D.; AOKI, S.; FUKAMACHI, Y.; ROQUET, F.; HINDELL, M.; USHIO, S.; OHSHIMA, K. I. Antarctic bottom water production from the Vincennes Bay Polynya, East Antarctica. Geophysical Research Letters, v. 41, n. 10, p. 3528-3534, 2014.

KUSAHARA, K.; HASUMI, H.; TAMURA, T. Modeling sea ice production and dense shelf water formation in coastal polynyas around East Antarctica. Journal of Geophysical Research: Oceans, v. 115, n. 10, p. 1-10, 2010.

KUSAHARA, K.; HASUMI, H.; WILLIAMS, G.D. Impact of the Mertz Glacier Tongue calving on dense water formation and export. Nature communications, v. 2, n. 10, p. 159, 2011.

KWOK, R.; COMISO, J. C.; MARTIN, S.; DRUCKER, R. Ross Sea polynyas: Response of ice concentration retrievals to large areas of thin ice. Journal of Geophysical Research: Oceans, v. 112, n. 12, p. 1-13, 2007.

LABROUSSE, S.; WILLIAMS, G.; TAMURA, T.; BESTLEY, S.; SALLÉE, J. B.; FRASER, A. D.; SUMNER, M.; ROQUET, F.; HEERAH, K.; PICARD, B.; GUINET, C.; HARCOURT, R.; MCMAHON, C.; HINDELL, M. A.; CHARRASSIN, J. B. Coastal polynyas: Winter oases for subadult southern elephant seals in East Antarctica. Scientific Reports, v. 8, n. 1, p. 1-15, 2018.

LANDSCHÜTZER, P.; GRUBER, N.; HAUMANN, F. A.; RÖDENBECK, C.; BAKKER,B. C.E.; HEUVEN,S. V.; HOPPEMA, M.; METZL, N.; SWEENEY, C.; TAKAHASHI, T.; TILBROOK, B.; WANNINKHOF, R. The reinvigoration of the Southern Ocean carbon sink. Science, v. 349, 12211224, 2015

LI, Y.; JI, R.; JENOUVRIER, S.; JIN, M.; STROEVE, J. Synchronicity between ice retreat and phytoplankton bloom in circum-Antarctic polynyas. Geophysical Research Letters, v. 43, n. 5, p. 2086-2093, 2016.

MADEC, G. \& IMBARD, M. A global ocean mesh to overcome the North Pole singularity. Climate Dynamics, v. 12, n. 6, p. 381-388, 1996.

MALPRESS, V.; Sophie Bestley, S.; Corney, S.; Welsford, D.; Labrousse, S.; Sumner, M.; Hindell, M. Bio-physical characterisation of polynyas as a key foraging habitat for juvenile male southern elephant seals (Mirounga leonina) in Prydz Bay, East Antarctica. PLoS ONE, v. 12, n. 9, p. 1-24, 2017.

MAQUEDA, M.A.M. Polynya Dynamics : a Review of
Observations and Modeling. Review of Geophysics, v. 42, n. RG1004, p. 1-37, 2004.

MARKUS, T. \& CAVALIERI, D.J. An enhancement of the NASA team sea ice algorithm. IEEE Transactions on Geoscience and Remote Sensing, v. 38, n. 3, p. 1387-1398, 2000.

MARTIN, S.; DRUCKER, R.; KOWK, R.; HOLT, B. Estimation of the thin ice thickness and heat flux for the Chukchi Sea Alaskan coast polynya from special sensor microwave/imager data, 1990-2001. Journal of Geophysical Research C: Oceans, v. 109, n. 10, p. 1990-2001, 2004.

MARTIN, S.; DRUCKER, R.S.; KWOK, R. The areas and ice production of the western and central Ross Sea polynyas, 1992-2002, and their relation to the B-15 and C-19 iceberg events of 2000 and 2002. Journal of Marine Systems, v. 68, n. 1-2, p. 201-214, 2007.

MASSOM, R.A.; HARRIS, P.T.; MICHAEL, K.J.; POTTER, M.J. The distribution and formative processes of latent-heat polynyas in East Antarctica. Annals of Glaciology, v. 27, p. 420-426, 1998.

MATEAR, R.J.; O’KANE, T.J.; JAMES, S. RISBEY, J.S.; CHAMBERLAIN, M. Sources of heterogeneous variability and trends in Antarctic sea-ice. Nature communications, v. 6, p. 8656, 2015.

MUNDY, C.J. \& BARBER, D.G. On the relationship between spatial patterns of sea-ice type and the mechanisms which create and maintain the North Water (NOW) polynya. Atmosphere - Ocean, v. 39, n. 3, p. 327-341, 2001.

NAKAYAMA, Y.; OHSHIMA, K.I.; MATSUMURA, Y.; FUKAMACHI, Y. A Numerical Investigation of Formation and Variability of Antarctic Bottom Water off Cape Darnley, East Antarctica. Journal of Physical Oceanography, v. 44, n. 11, p. 2921-2937, 2014.

NIHASHI, S. \& OHSHIMA, K.I. Circumpolar mapping of antarctic coastal polynyas and landfast sea ice: Relationship and variability. Journal of Climate, v. 28, n. 9, p. 3650-3670, 2015.

NIHASHI, S.; OHSHIMA, K. I.; TAMURA, T.; YASUSHI FUKAMACHI, Y.; SAITOH, S. Thickness and production of sea ice in the Okhotsk Sea coastal polynyas from AMSR-E. Journal of Geophysical Research: Oceans, v. 114, n. 10, p. $1-15,2009$.

NÚÑEZ-RIBONI, I. \& FAHRBACH, E. Seasonal variability of the Antarctic Coastal Current and its driving mechanisms in the Weddell Sea. Deep-Sea Research Part I: Oceanographic Research Papers, v. 56, n. 11, p. 1927-1941, 2009.

O'BRIEN, P.E.; HARRIS, P.T.; POST, A.L.; NEAL, W YOUNG, N.W. Chapter 18 East Antarctic continental shelf: Prydz Bay and the Mac.Robertson Land Shelf. Geological Society, London, Memoirs, v. 41, n. 1, p. 241-254, 2014.

OHSHIMA, K.I. Surface Heat Budget of the Sea of Okhotsk during 1987 - 2001 and the Role of Sea Ice on it. Journal of the Metereorological Society os Japan, v. 81, n. 4, p. 653677, 2003.

OHSHIMA, K.I.; FUKAMACHI, Y.; WILLIAMS, G.D.; NIHASHI, S.; ROQUET, F.; KITADE, Y.; TAMURA, T.; HIRANO, D.; HERRAIZ-BORREGUERO, L.; FIELD, I.;HINDELL, M.; AOKI, M.; WAKATSUCH, M. Antarctic Bottom Water production by intense sea-ice formation in the Cape Darnley polynya. Nature Geoscience, v. 6, n. 3, p. 235240, 2013.

ORSI, A.H.; JOHNSON, G.C.; BULLISTER, J.L. Circulation, mixing, and production of Antarctic Bottom Water. Progress in Oceanography, v. 43, n. 1, p. 55-109, 1999.

PALMER, M.D.; ROBERTS, C.D.; BALMASEDA, M.; CHANG, Y.S.; CHEPURIN, G.; FERRY, N.; FUJII, Y.; GOOD, S.A.; GUINEHUT, S.; HAINES, K.; HERNANDEZ, F.; KÖHL, A.; LEE, T.; MARTIN, M.J.; MASINA, S.; MASUDA, S.; PETERSON, K.A.; STORTO, A.; TOYODA, T.; VALDIVIESO, M.; VERNIERES, G.; WANG, O.; XUE, Y. OCEAN HEAT CONTENT VARIABILITY AND 
CHANGE IN AN ENSEMBLE OF OCEAN REANALYSES. Climate Dynamics, v. 49, n. 3, p. 909-930, 2017.

PARENT, S.T.L; KLINCK, J.M.; DINNIMAN, M.S. Impact of local winter cooling on the melt of Pine Island Glacier, Antarctica. Journal of Geophysical Research: Oceans, v. 120, p. 6718-6732, 2015.

PARMIGGIANI, F. Fluctuations of Terra Nova Bay polynya as observed by active (ASAR) and passive (AMSR-E) microwave radiometers. International Journal of Remote Sensing, v. 27, n. 12, p. 2459-2467, 2006.

PAUL, S.; WILLMES, S.; HEINEMANN, G. Long-term coastal-polynya dynamics in the southern Weddell Sea from MODIS thermal-infrared imagery. The Cryosphere, v. 9, p. 2027-2041, 2015.

SCHMIDTKO, S.; KAREN, J.; HEYWOOD, K.J.; THOMPSON, A.F.; SHIGERU AOKI, S. Multidecadal warming of Antarctic waters. Science, v. 346, n. 6214, p. 1227-1231, 2014.

SHADWICK, E.H.; TILBROOK, B.; WILLIAMS, G.D. Carbonate chemistry in the Mertz Polynya (East Antarctica): Biological and physical modification of dense water outflows and the export of anthropogenic CO2. Journal of Geophysical Research: Oceans, v. 119, n. 1, p. 1-14, 2014.

SILVANO, A.; RINTOUL, S.R.; PEÑA-MOLINO, B.; HOBBS, W.R.; VAN WIJK, E.; AOKI, S.; TAMURA,T.; WILLIAMS, G.D. Freshening by glacial meltwater enhances melting of ice shelves and reduces formation of Antarctic Bottom Water. Science Advances, v. 4, n. 4, p. eaap9467, 2018.

SMITH, S.D.; MUENCH, R.D.; PEASE, C.H. Polynyas and leads: An overview of physical processes and environment. Journal of Geophysical Research, v. 95, n. C6, p. 9461, 1990.

TA.D. Closure of the global overturning circulation through the Indian, Pacific and Southern Oceans: schematics and transports. Oceanography, special issue, 2013.

TAMURA, T. \& OHSHIMA, K.I. Mapping of sea ice production in the Arctic coastal polynyas. Journal of Geophysical Research: Oceans, v. 116, n. 7, p. 1-5, 2011

TAMURA, T.; OHSHIMA, K.I.; MARKUS, T.; CAVALIERI, D.J.; NIHASHI, S.; HIRASAWA, N. Estimation of thin ice thickness and detection of fast ice from SSM/I data in the Antarctic Ocean. Journal of Atmospheric and Oceanic Technology, v. 24, n. 10, p. 1757-1772, 2007.

TAMURA, T.; OHSHIMA, K.I.; FRASER, A.D.; GUY, D.; WILLIAMS, G.D. Sea ice production variability in Antarctic coastal polynyas. Journal of Geophysical Research: Oceans, 2016.

TAMURA, T.; OHSHIMA, K. I.; NIHASHI, S. Mapping of sea ice production for Antarctic coastal polynyas. Geophysical Research Letters, v. 35, 2008.

TAMURA, T.; WILLIAMS, G.D.; FRASER, A.D.; OHSHIMA. K.I. Potential regime shift in decreased sea ice production after the Mertz Glacier calving. Nature Communications, v. 3, n. May, p. 826, 2012.
TIMMERMANN, R.; GOOSSE, H.; MADEC, G.; FICHEFET, T.; ETHE, C.; DULIÈRE, V. On the representation of high latitude processes in the ORCA-LIM global coupled sea iceocean model. Ocean Modelling, v. 8, n. 1-2, p. 175-201, 2005.

TURNER, J.; HOSKING, J. S.; MARSHALL, G. J.; PHILLIPS, T.; BRACEGIRDLE, T. J.Antarctic sea ice increase consistent with intrinsic variability of the Amundsen sea low. Climate Dynamics, v. 46, n. 7-8, p. 2391-2402, 2016.

TURNER, J.;PHILLIPS, T.; MARSHALL, G. J.; POPE, J. O.; BRACEGIRDLE, T. J.; DEB, P. Unprecedented springtime retreat of Antarctic sea ice in 2016. Geophysical Research Letters, v. 44, n. 13, p. 6868-6875, 2017.

TURNER, J.; COMISO, J.C.; MARSHALL, G.J.; LACHLAN COPE, T.A.; BRACEGIRDLE, T.J.; MAKSYM, T.; MEREDITH, M. P.; WANG, Z.; ORR, A. Non-annular atmospheric circulation change induced by stratospheric ozone depletion and its role in the recent increase of Antarctic sea ice extent. Geophysical Research Letters, v. 36, n. 8, p. 1-5, 2009.

UOTILA, P.; ZHAORU, Z.; VIHMA, T.; STÖSSEL, A. The role of wind forcing from operational analyses for the model representation of Antarctic coastal sea ice. Ocean Modelling, v. 94, n. October, p. 95-111, 2015.

WILLIAMS, G.D.; AOKI, S.; JACOBS, S.S.; RINTOUL, S.R.; TAMURA, T.; BINDOFF, N.L. Antarctic Bottom Water from the Adélie and George V Land coast, East Antarctica (140 $149^{\circ}$ E). Journal of Geophysical Research, v. 115, p. 1-29, 2010.

WILLIAMS, G.D.; HERRAIZ-BORREGUERO, L.; ROQUET, F.; TAMURA, T.; OHSHIMA, K. I.; FUKAMACHI, Y.; FRASER, A.D.; GAO, L.; CHEN, H.; MCMAHON, C.R.; HARCOURT, R.; HINDELL, M. The suppression of Antarctic bottom water formation by melting ice shelves in Prydz Bay. Nature Communications, v. 7, n. 6, p. 1-9, 2016.

WMO. Sea-Ice Nomenclature, terminology, codes and illustrated glossary. Sea Ice Nomenclature, v. 1, n. 259, p. 1121,1970

WUNSCH, C.; PONTE, R.M.; HEIMBACH, P. Decadal trends in sea level patterns: 1993-2004. Journal of Climate, v. 20, n. 24, p. 5889-5911, 2007.

ZWALLY, H.J.; COMISO, J.C.; PARKINSON, C.L.; CAVALIERI, D. J.; GLOERSEN, P. Variability of Antarctic sea ice 1979-1998. Journal of Geophysical Research, v. 107, n. C5, p. 3041, 2002. 